\title{
Review of Aroma Formation through Metabolic Pathways of Saccharomyces cerevisiae in Beverage Fermentations
}

\author{
Marissa B. Hirst ${ }^{1}$ and Chandra L. Richter ${ }^{1 *}$
}

\begin{abstract}
Fermentation has historically played an important role in the production of several commodities such as bread and alcoholic beverages. Today, fermentation is also used to produce specific flavor compounds in multiple industries. Flavor compounds are secondary metabolites produced during fermentation in addition to primary metabolites, such as ethanol. Secondary metabolism is influenced by fermentable carbon, nitrogen makeup, and the fermentation environment. A better understanding of how these variables affect the physiology of yeast strains to produce flavor compounds may improve several industrial commodities. To this end, systems biology represents an attractive strategy for studying the complex dynamics of secondary metabolism. Although applying systems biology methods to winemaking or brewing is not a new concept, directly linking -omics data with the production of flavor compounds represents a novel approach to improving flavor production in fermentation. Thus far, the bulk of the work in which systems biology methods have been applied to fermentation has relied heavily on laboratory strains of Saccharomyces cerevisiae that lack metabolism-relevant genes present in industrial yeast strains. Therefore, investigations of industrial strains with systems biology approaches will provide a deeper understanding of secondary metabolism in industrial settings. Ultimately, integrating multiple -omics approaches will lay the foundation for predictive models of $S$. cerevisiae fermentation and optimal flavor production.
\end{abstract}

Key words: beer, cider, fermentation, Saccharomyces cerevisiae, systems biology, wine

In the earliest fermentations, bread and alcohol were produced mainly by spontaneous inoculation with wild yeast from the environment. Starting in the late 19th century, industrial fermentations were purposefully inoculated with commercially produced yeast strains. These strains were propagated and sold based on how efficiently they produced the desired commodity, resulting in the selection of yeast strains for specific industries (Richter and Pugh 2012). Today, fermentation is used to produce specific metabolites used in the beverage, food, cosmetic, medical, and biofuel industries (Vandamme and Soetaert 2002). Therefore, research aimed at maximizing the quality and efficiency of metabolite production will provide significant technical and economic benefits to fermentation industries. This review will describe the metabolic pathways involved in the formation of aroma through fermentation.

In 2013, the worldwide flavor and fragrance market was estimated to be worth US $\$ 23.9$ billion (Venkataraman et al. 2014). More than 100 flavor molecules are produced via the conversion of natural precursors through microbial enzymes or by microbial fermentation involving native yeast strains or strains with gene modifications (Vandamme and Soetaert

${ }^{1}$ E \& J Gallo Winery, Department of Viticulture, Chemistry and Enology, P.O. Box 1130, Modesto, CA 95353.

*Corresponding author (Chandra.Richter@ejgallo.com; tel: 209 341-8429; fax: 2093414541 )

Acknowledgments: This work was supported by the E \& J Gallo Winery. This review would not have been possible without the support of Tom Smith, Vice President of Winegrowing at E \& J Gallo Winery, and Nick Dokoozlian, Vice President of Viticulture, Chemistry, and Enology at E \& J Gallo Winery.

Manuscript submitted Oct 2015, revised Apr 2016, accepted Jul 2016

Copyright ( $\odot 2016$ by the American Society for Enology and Viticulture. All rights reserved.

doi: 10.5344/ajev.2016.15098
2002). Compounds produced by yeast fermentation include glycerol, aldehydes, alcohols, propanediol, organic acids, isoprenoids, esters, and steroids.

\section{Generation of Aroma through Yeast Metabolism}

Although Saccharomyces cerevisiae is the most commonly used fermentation organism, many other genera of yeast can also be used in this process, including Saccharomycodes, Schizosaccharomyces, Candida, Torulaspora, Cryptococcus, Debaryomyces, Issatchenkia, Pichia, Kluyveromyces, Metschnikowia, Hanseniaspora (Kloeckera), Rhodotorula, Brettanomyces (Dekkera), and Zygosaccharomyces (Vandamme and Soetaert 2002). Yeast strains or species are selected according to the aesthetic or flavor components they produce in commercial food and beverage products. "Flavor" will be defined here as the volatile and nonvolatile compounds related to aroma and mouthfeel, respectively. Nonvolatile compounds include glycerol, mono- and polysaccharides, phenolics, and organic acids; volatile compounds include alcohols, aldehydes, esters, dicarbonyls, short- and mediumchain fatty acids, methyl ketones, lactones, phenolic and sulfur compounds, and terpenes (Moreno-Arribas and Polo 2009). The many flavor metabolites that yeasts produce during fermentation (Figure 1) are generated de novo or by the transformation and volatilization of precursor compounds in the starting material (fruit juice, hops, grains, etc.) (Lambrechts and Pretorius 2000).

The metabolism of fermenting $S$. cerevisiae comprises two stages: primary and secondary metabolism. Primary metabolism is essential for growth, cell division, and survival, producing metabolites such as ethanol, glycerol, acetaldehyde, and acetic acid (Styger et al. 2011). Secondary metabolism is 
nonessential for growth and produces small molecules that include the fusel alcohols, esters, carbonyls, sulfur compounds, thiols, and terpenoids (Figure 1) (Styger et al. 2011). Secondary metabolites can contribute to the organoleptic properties of food and beverage products and are important commodities for several industries. Secondary metabolism is greatly influenced by fermentable carbon, nitrogen makeup, and the fermentation environment (Henschke and Jiranek 1993, Verstrepen et al. 2004). A better understanding of how these variables influence production of yeast aroma compounds can be used to improve beverage, food, perfume, and cosmetic products.

\section{Regulation of Secondary Metabolism}

Fusel alcohols. Fusel alcohols are the most abundant volatile components produced during fermentation and contribute to essential aroma and flavors in fermented beverages and food (Hazelwood et al. 2008). Fusel alcohols include propanol, isoamyl alcohol, isobutanol, active amyl alcohol, 2-phenylethanol, and tyrosol (Swiegers and Pretorius 2005). Fusel alcohols form through the Ehrlich pathway or via central carbon metabolism (Figure 2A). The first step in the Ehrlich pathway is a transamination reaction between an amino acid and 2-oxoglutarate (Sentheshanmuganathan 1960). The transamination step converts the amino acid to an $\alpha$-keto acid, which may also be supplied by central carbon metabolism (Sentheshanmuganathan 1960). Subsequently, multiple pyruvate decarboxylases catalyze the conversion of the $\alpha$-keto acid to a branched-chain aldehyde (Sentheshanmuganathan 1960). Last, an alcohol dehydrogenase catalyzes the NADH-dependent final step that reduces the aldehyde to a fusel alcohol (Figure 2A) (Hazelwood et al. 2008). Not all enzymes involved in the catalysis of this pathway are known. One of the difficulties in elucidating

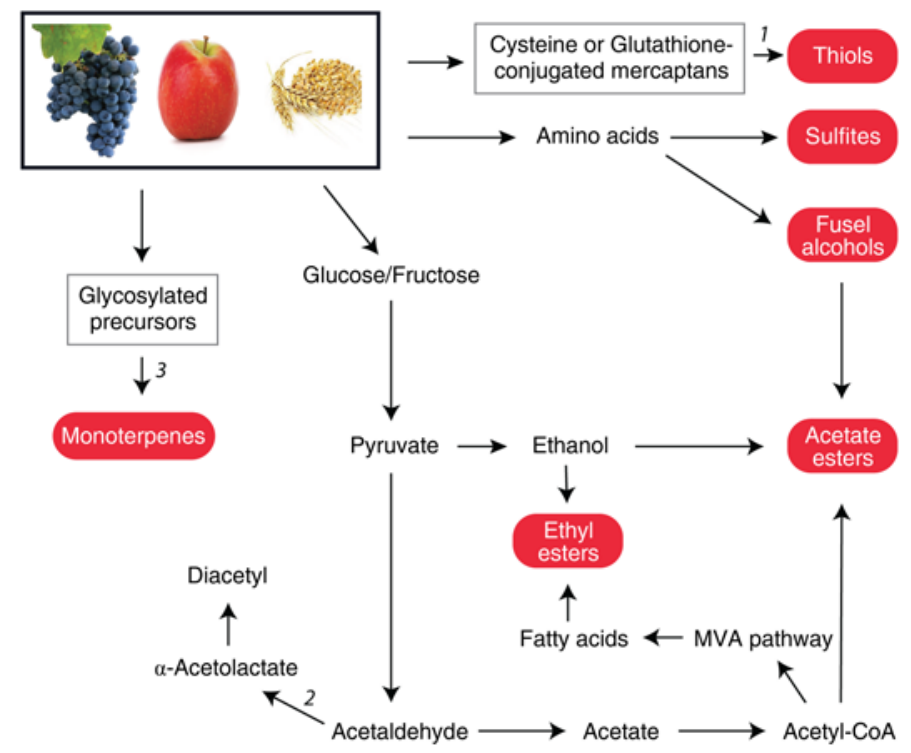

Figure 1 Aroma compounds (red boxes) and nonvolatile precursor metabolites (boxes with gray lines) produced by Saccharomyces cerevisiae during fermentation. Cysteine or glutathione-conjugated mercaptans are present in grapes and hops; glycosylated precursors are present in fruit but not in grain. Italicized numbers above or next to arrows represent enzymes catalyzing the reactions. 1: Irc7 and Str3; 2: Ilv2; and 3: Exg1. specific proteins necessary for fusel alcohol production is a large degree of redundancy in the yeast genome. The presence of multiple aminotransferase, decarboxylase, and alcohol dehydrogenase genes poses challenges in traditional genetic approaches to studying fusel production.

Fusel alcohols can have positive or negative sensory impacts, depending on their concentration. Fusel alcohol concentrations of $400 \mathrm{mg} / \mathrm{L}$ or greater may result in a pungent, solvent-like aroma in wine, whereas concentrations of less than $300 \mathrm{mg} / \mathrm{L}$ are often described as imparting a desirable fruity characteristic (Swiegers and Pretorius 2005). In ciders, high concentrations of fusel alcohols, particularly 2-phenylethanol, are important contributors to the typical cider flavor (Beech 1972). Propanol, butanol, and isobutanol are described

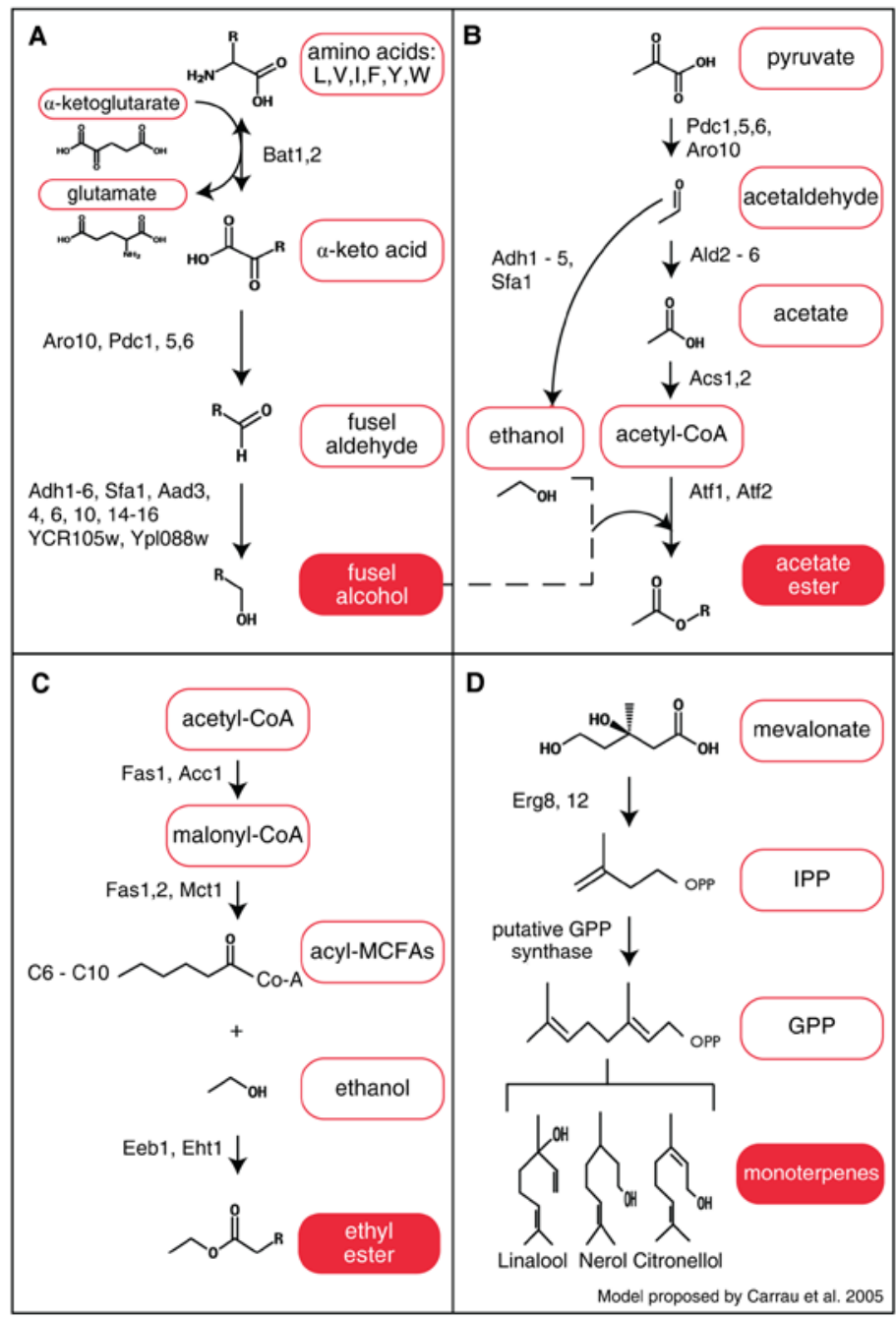

Figure 2 Metabolic pathways leading to the formation of aroma compounds (Carrau et al. 2005) produced by Saccharomyces cerevisiae during fermentation. (A) The fusel alcohol pathway (via the Ehrlich pathway) transforms an amino acid to an alcohol in multiple steps. The amino acids in this pathway include leucine (L), valine (V), isoleucine (I), phenylalanine $(\mathrm{F})$, tyrosine $(\mathrm{Y})$, and tryptophan $(\mathrm{W})$. (B, C) The formation of esters requires an alcohol. The alcohol can be either ethanol or a fusel alcohol. (B) The acetate ester pathway. (C) The ethyl-ester synthesis pathway. (D) In vivo synthesis of monoterpenes through the mevalonate pathway. Red boxes: volatile aroma compounds. 
as having an alcoholic odor, whereas active amyl alcohol and isoamyl alcohol are described as having a marzipan-like or banana aroma (Lambrechts and Pretorius 2000). Tyrosol and 2-phenylethanol impart honey-like and floral aromas, respectively (Lambrechts and Pretorius 2000). In appropriate concentrations, fusel alcohols impart a beneficial complexity and also serve as precursors for the formation of acetate esters.

Acetate and ethyl esters. Esters contribute to the floral and fruity characteristics associated with wines and beer and are generated by the esterification of alcohol and acids at low $\mathrm{pH}$ (Saerens et al. 2010). The reaction requires an alcohol molecule, acetyl-CoA, an ester-synthesizing enzyme, and adenosine triphosphate (ATP; Figure 2B and 2C) (Saerens et al. 2010). Both the production and degradation of esters are tightly regulated.

The two ester types produced during fermentation are acetate and ethyl esters. Acetate esters result from the esterification of acetyl-CoA and an alcohol (Figure 2B). These acetates include ethyl acetate, isoamyl acetate, 2-methylbutyl acetate, and phenylethyl acetate, and are described as having banana, apple, fruit, and aromatic sweetness, respectively (Saerens et al. 2010). Ethyl acetate is the most common acetate ester in fermentations, primarily because of the large quantities of ethanol in these metabolic processes and the more reactive nature of primary alcohols (Saerens et al. 2010).

The regulation of acetate ester production is primarily controlled by the expression of two alcohol acetyl transferases (AATase), Atflp and Atf2p (Figure 2B) (Yoshimoto et al. 1998). Atflp has the greatest AATase activity; introducing multiple copies of ATF1 into laboratory strains results in increased production of acetate esters (Verstrepen et al. 2003). In contrast, deletion of Atflp significantly decreases acetate ester concentration (Verstrepen et al. 2003). The production of acetate esters is also substrate dependent, relying on the availability of fusel alcohols (Lilly et al. 2006).

The second group, the ethyl esters, is composed of ethanol and a medium-chain fatty acid (MCFA) (Figure 2C) (Saerens et al. 2010). Ethyl esters include ethyl butanoate, ethyl hexanoate, ethyl octanoate, and ethyl decanoate (Saerens et al. 2010). These ethyl esters vary in their sensorial attributes, but descriptions include apple, fruit, strawberry, pear, and aniseed (Saerens et al. 2010). During the late exponential growth phase, MCFA intermediates are released prematurely from the cytoplasmic fatty acid synthase (FAS) complex, and this release triggers ester synthesis (Taylor and Kirsop 1977). The MCFAs are activated by coenzyme A and are esterified in conjunction with ATP, ethanol, and enzymes (Figure 2C) (Saerens et al. 2010).

Experimental evidence points to three regulatory pathways responsible for the release of MCFAs and subsequent production of ethyl esters: (1) decreased acetyl-CoA carboxylase activity; (2) upregulation of the fatty acid biosynthesis genes FAS1, FAS2, EEB1, and EHT1; and (3) concentrations of MCFAs (Saerens et al. 2006, Dufour et al. 2008). Inhibition of acetyl-CoA carboxylase initiates the release of MCFAs from the FAS complex (Dufour et al. 2008). Deletion of EEBI and $E H T 1$ decreases the production of ethyl esters; however, over- expression of both enzymes does not result in an increase in these esters (Saerens et al. 2006). Several genes have been implicated in the production and degradation of esters; however, direct linkages between precursors, enzyme activity, substrate concentration, and ester production have not yet been identified, particularly not for ethyl esters.

Carbonyls: Diacetyl. Diacetyl is described as having a toasty, butterscotch, and nutty aroma, but at high concentrations, it smells like rancid butter (Swiegers and Pretorius 2005). Yeast cells may synthesize diacetyl during fermentation; in wine, however, diacetyl is predominantly produced by lactic acid bacteria (Laurent et al. 1994). Diacetyl is formed extracellularly by chemically driven decarboxylation of $\alpha$-acetolactate. $\alpha$-Acetolactate is synthesized as an intermediate in the 2-ketoisovalerate pathway or produced from acetaldehyde by Ilv2p (Figure 1) (Suomalainen and Ronkainen 1968). Diacetyl is subsequently converted to acetoin, followed by conversion of acetoin to 2,3-butanediol, both of which have a higher sensory threshold and therefore less sensory impact (Swiegers and Pretorius 2005).

Sulfur compounds. Sulfur-containing compounds have a very low sensory detection threshold and are often described as having a cabbage, rotten egg, or onion aroma (Rauhut 1993). There are five categories of sulfur compounds: sulfides, polysulfides, thiols, thioesters, and heterocyclic compounds (Rauhut 1993). Aromatic sulfur compounds originate from sulfur-containing fungicides or from the degradation of sulfur-containing amino acids during fermentation; however, the pathways responsible for these processes have not been fully elucidated (Rauhut 1993).

Hydrogen sulfide $\left(\mathrm{H}_{2} \mathrm{~S}\right)$ contributes to one of the primary sulfur off-notes; it is produced during fermentation by the sulfate-reduction sequence pathway. In this pathway, sulfate is taken up from the external medium via a sulfate permease (Sullp or Sul2p) and reduced to sulfite and then to sulfide via Met5p and Met10p, respectively (Rauhut 1993, Spiropoulos and Bisson 2000). In the presence of nonlimiting cysteine or methionine concentrations, sulfide combines with $O$-acetylserine or $O$-acetylhomoserine to form homocysteine (Swiegers and Pretorius 2005). Under methionine- and cysteinelimited conditions, $O$-acetylserine and $O$-acetylhomoserine are also limited, resulting in excess sulfide that is converted to $\mathrm{H}_{2} \mathrm{~S}$ (Thomas and Surdin-Kerjan 1997).

Agricultural use of elemental sulfur, pantothenate deficiency, and high concentrations of threonine can also affect $\mathrm{H}_{2} \mathrm{~S}$ production during fermentation (Spiropoulos and Bisson 2000). Hydrogen sulfide is very reactive, often resulting in additional off-notes in wine. One of the more common offnotes produced as a result of $\mathrm{H}_{2} \mathrm{~S}$ reactivity is ethanethiol, which is formed when $\mathrm{H}_{2} \mathrm{~S}$ reacts with ethanol (Spiropoulos and Bisson 2000). Hydrogen sulfide can be removed from wine by copper stripping or nitrogen aeration, but these treatments may also remove valuable aroma compounds (Swiegers and Pretorius 2005).

In contrast to other sulfur compounds, volatile thiols have beneficial aroma characteristics. The aromas of volatile thiols are described as boxwood, passion fruit, black currant, 
or grapefruit (Tominaga et al. 1998). These compounds are often $S$-cysteine- or glutathione-bound in grape or hops and are released during the fermentation (Tominaga et al. 1998). Irc $7 p$ and Str3p cleave the carbon-sulfur bond between cysteine and the thiol to release the aroma (Figure 1) (Tominaga et al. 1998).

The three most impactful volatile thiols are 4-mercapto4-methylpentan-2-one (4MMP), 3-mercaptohexan-1-ol (3MH), and 3-mercaptohexyl acetate (3MHA). Harsch et al. (2013) identified (E)-2-hexenal and its corresponding alcohol, (E)2-hexen-1-ol, as precursors to the formation of 3MH (Harsch et al. 2013). This work established the potential for enhancing $3 \mathrm{MH}$ and $3 \mathrm{MHA}$ in the presence of a sulfur donor and (E)-2-hexenal and (E)-2-hexen-1-ol, opening the door to the possibility of using yeast to enhance production of positive aroma thiols (Figure 3) (Harsch et al. 2013).

Terpenoids. Terpenoids are a class of aroma compounds that define varietal characteristics in fruit and hops, producing aromas described as rose, geranium, and floral (Swiegers and Pretorius 2005). Terpenoids are essential flavor components in many commercial products, including wine, beer, food additives, perfumes, and cosmetics. Optimizing the production of volatile terpenes during yeast fermentation is a major goal in industrial fermentation.

Monoterpenoids are produced by the precursor geraniol pyrophosphate (GPP) in plants and some fungi (King and

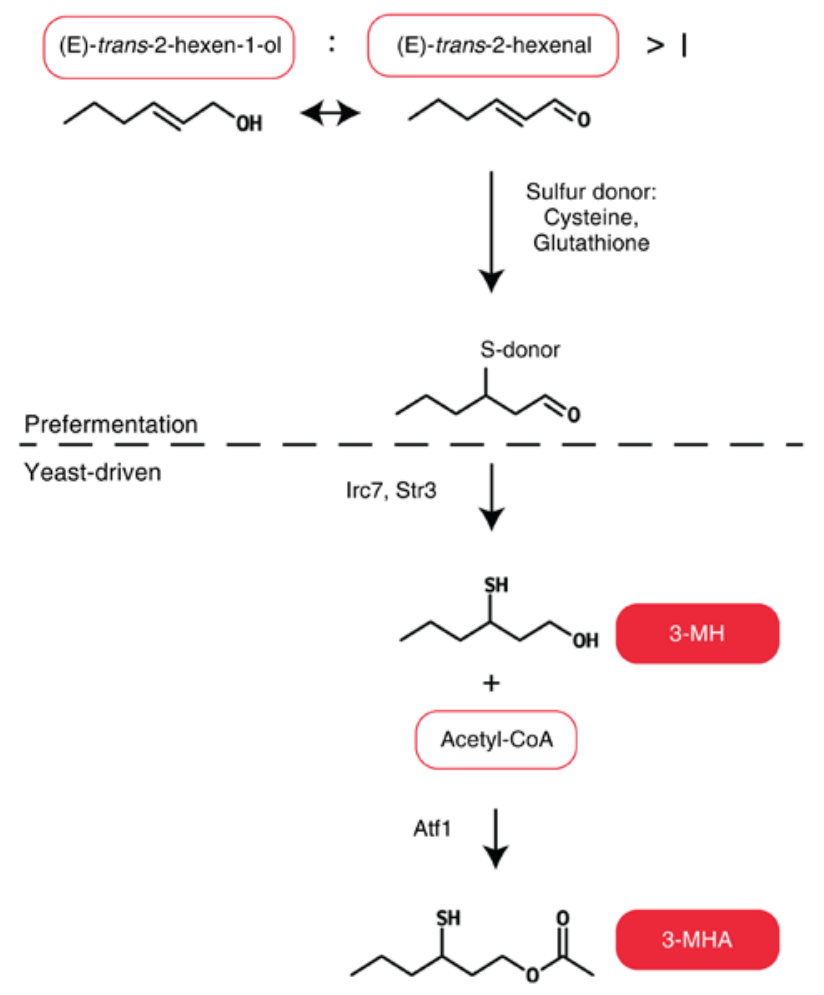

Figure 3 The predicted pathway leading to the production of the aroma thiols 3-mercaptohexan-1-ol (3-MH) and 3-mercaptohexyl acetate (3MHA) (adapted from Harsch et al. 2013). When the six-carbon (C6) precursors, (E)-trans-2-hexen-1-ol and (E)-trans-2-hexanal have a relative ratio greater than one and a sulfur donor is present, the reaction is driven to produce 3-MH and 3-MHA. When fermentation begins, yeast cells release the aroma thiol 3-MH; acetylation of 3-MH results in 3-MHA.
Richard Dickinson 2000). Vitis vinifera (grapevines) and Humulus lupulus (hops) synthesize monoterpenoids such as geraniol, linalool, nerol, and citronellol (Swiegers and Pretorius 2005). Terpenoids are found in both free and bound forms, with the bound form being more prevalent (Swiegers and Pretorius 2005). Yeast glycosidase enzymes release and volatilize these aroma compounds during fermentation (Figure 1) (Gunata et al. 1988).

The enzymatic release of monoterpenes is a one- or twostep process, depending on the sugar bound to the precursor glucoside (i.e., a mono- or disaccharide) (Gunata et al. 1988). For disaccharide glycosides, the first step involves the release of the terminal sugar via an arabinofuranosidase, rhamnopyranosidase, or apiofuranosidase, followed by $\beta$-glucosidase cleavage releasing the terpenoid (Gunata et al. 1988). Wine strains overexpressing cell wall protein Exglp produce elevated levels of terpenes in both synthetic media and grape must (Gil et al. 2005).

Terpenoid production has primarily been attributed to hydrolysis of glycosidic linkages; however, S. cerevisiae is capable of producing monoterpenes through the mevalonic acid pathway (Figure 2D). Carrau et al. (2005) showed that $S$. cerevisiae and Hanseniaspora uvarum can produce terpenes in chemically defined medium lacking grape juice, terpenes, and glycoconjugates. This work revealed that linalool and $\alpha$-terpineol were produced by $S$. cerevisiae under microaerobic and high-assimilable-nitrogen conditions in synthetic media. Carrau et al. (2005) have predicted an alternative pathway for monoterpene synthesis (Figure 2D); in this pathway, the monoterpenes are formed de novo in the mitochondria and linked to leucine catabolism and a novel GPP synthase. A linkage between leucine catabolism and isoprenoid metabolism has been studied and established in the fungus Aspergillus nidulans, providing precedence for this alternative pathway (Rodríguez et al. 2004).

\section{Impact of Starting Materials on Production of Flavor Components}

Fermentation requires a carbon source (sugar), nitrogen (ammonia and amino acids), water, and yeast. The aromatic by-products produced during fermentation can vary drastically, depending on the starting material (fruit, grain, or extract), the concentrations of carbon and nitrogen, and the yeast strain used (Henschke and Jiranek 1993, Verstrepen et al. 2004, Richter and Pugh 2012). Because of this variability, industrial fermentation has been optimized for the desired commodity product. For example, fermentations for alcoholic beverage products seek to enhance specific flavor compounds. Fruity and flowery esters are desirable in wine (Moreno-Arribas and Polo 2009) but may be less desirable in certain beer styles. Fusel alcohol levels exceeding $300 \mathrm{mg} / \mathrm{L}$ are sought after in hard ciders (Beech 1972) but may produce off-notes in wine.

The starting material plays a crucial role in affecting the final flavor profile of the wine, beer, cider, or sake. For example, free terpenoid compounds in grapes impart specific aroma characteristics and vary depending on the grape varietal (Conde et al. 2007). Muscat grapes have the highest 
concentration of free monoterpenes, providing a distinct floral aroma (Conde et al. 2007). In contrast, the monoterpenes in Cabernet Sauvignon, Sauvignon blanc, Merlot, Shiraz, and Chardonnay are typically below the sensory threshold (Conde et al. 2007).

Apples impart unique flavor components to cider because they contain high concentrations of phenolics and have high acidity (Barker and Burroughs 1953, Beech 1972). The aromatic profile produced from apple juice concentrate (apple aroma essence) primarily comprises six compounds, including ethyl-2-methyl butyrate, 1-hexanal, trans-2-hexanal, ethyl acetate, 1-butanol, and cis-3-hexenol (Beech 1972). The sensory profile of cider made from freshly pressed apples differs from that made from concentrate. Fresh apple juice has a lower overall concentration of the apple aroma essence, which is created through evaporation, condensation, and multiple rounds of concentration of fresh juice (Beech 1972). Another important class of compounds crucial for cider flavor is the fusel alcohols (Beech 1972). Yeast produce fusel alcohols during fermentation, and fusel alcohol levels are influenced by apple variety, juice treatment prior to fermentation, yeast strain, storage condition, and carbon and nitrogen content of the juice or concentrate (Beech 1972).

Beer requires four essential ingredients: water, hops, yeast, and grain, all of which can affect the final flavor profile. The cereal grain used to generate malt for brewing is typically barley but may also include wheat, rye, oats, sorghum, or millet (Briggs et al. 2004). Nonbarley malts impart distinct flavor characteristics to beer and fermented beverages. For example, beers produced from 50\% malt and 50\% polished sorghum have lower concentrations of isobutanol (alcoholic aroma), 2-methylbutanol (marzipan), and dimethyl sulfide (cabbage and gasoline), but have higher levels of 1-propanol (stupefying aroma) and diacetyl (butterscotch, butter) than beers brewed with $100 \%$ barley malt (Dale et al. 1990). Additionally, batches of malted oats used in place of barley malt produce lower-alcohol, higher-pH beers with lower overall concentrations of fusel alcohols and of the esters ethyl acetate and isoamyl acetate (Klose et al. 2011). The concentration of vicinal diketones, such as 2,3-pentanedione and diacetyl, were reported to be above the sensory threshold in oat malt beers and below this threshold in barley malt beers, and impart an overall yogurt and berry-like aroma to beer made solely with oat malt (Klose et al. 2011). In contrast, beers brewed with $100 \%$ barley malt were reported to have an apple-like flavor (Klose et al. 2011). These studies revealed that altering the grain source drastically changes the flavor components of the final beer, which can be exploited to target different flavor profiles during fermentation.

Carbon source and its impact on fermentation. In industrial fermentations, sugars provide essential carbon. The concentration of usable sugars will vary depending on the starting material (fruit or grain) (Figure 4A). Wine, cider, and beer will vary in their sensorial characteristics in part because of the different sugars and relative ratios of those sugars in the starting material (Figure 4A). Although many sugars may be available, $S$. cerevisiae preferentially utilizes glucose and sucrose by downregulating genes involved in the uptake of alternative carbon sources (Figure 4B) (De Deken 1966). Glucose also limits the uptake of fructose by $S$. cerevisiae because these two sugars are taken up into the cell via the same transporters (Reifenberger et al. 1997). As fructose is sweeter than glucose, fermentations with high residual fructose will affect the overall sweetness and flavor of the final product.

High concentrations of glucose and sucrose also downregulate the stress signaling pathway in $S$. cerevisiae by triggering the activation of the Ras/cyclic-adenosine monophosphate (cAMP)/protein kinase A (PKA) pathway, which decreases stress resistance of yeast cells (Figure 4B) (Rolland et al. 2000). Such downregulation of the stress pathways can result in sluggish fermentations and cell autolysis. Cell autolysis increases "goat-like" flavors by releasing fatty acids into the fermentation media and decreases fruity or floral flavors through ester hydrolysis (Anderson and Kirsop 1974).

Once glucose and sucrose have been depleted by $S$. cerevisiae, catabolite derepression occurs in the yeast cells, allowing for the uptake of alternative sugars (Figure 4C). The transition time to catabolite derepression will vary with the starting concentration of glucose in the must or malt. For example, derepression occurs more rapidly in wort than in fruit must because of the lower concentration of glucose and sucrose in the former (Figure 4A). Carbon source has a huge impact on beer aroma; specifically, worts with high glucose and fructose concentrations have higher levels of acetate esters (Anderson and Kirsop 1974). This can be problematic for high-gravity (high-sugar) brewing, as the resulting beers are overly fruity and chemical. For example, ethyl acetate and isoamyl acetate concentrations were four-fold higher in highgravity beers than in low-gravity beers (Anderson and Kirsop 1974). Worts supplemented with maltose syrups, however, had lower ethyl acetate $(10 \%)$ and isoamyl acetate concentrations (40\%) (Younis and Stewart 1999), suggesting that high-gravity beers can be brewed with higher concentrations of maltose to reduce the overall concentration of acetate esters (Younis and Stewart 2000). These studies also highlight the linkage between carbon source and flavor profiles. These processes are likely transcriptionally and translationally controlled through yeast metabolism (Figure 4B and 4C).

Impact of nitrogen on fermentation. In addition to carbon, nitrogen is important for growth, cell division, and secondary metabolism of yeast cells. The two forms of yeast assimilable nitrogen are primary amino acids and ammonium (Henschke and Jiranek 1993). In nitrogen-rich conditions, $S$. cerevisiae cells are under nitrogen catabolite repression (NCR) controlled by the target of rapamycin (TOR) complex (Figure 4B) (Hardwick et al. 1999). NCR downregulates genes encoding permeases and enzymes needed for the uptake and transport of poor nitrogen sources (Figure 4B). TOR1p is also responsible for regulating glucose activation, glycolysis, and the TCA cycle, creating a tight link between carbon and nitrogen uptake in the cell (Hardwick et al. 1999).

The NCR-induced downregulation of genes is abolished as nitrogen conditions shift from rich to poor, forcing $S$. cerevisiae to utilize alternative sources of nitrogen (Figure 
4C). Depending on the starting material (fruit or grain) and the yeast strain, amino acids will be taken up by order of preference. Typically, glutamic acid, aspartic acid, asparagine, glutamine, serine, threonine, lysine, and arginine are preferred amino-acid sources for S. cerevisiae (Jones and Pierce 1964).

The production of secondary metabolites is tightly linked to nitrogen source. Fusel alcohols may be formed through the catabolic Ehrlich pathway (Sentheshanmuganathan 1960, Hazelwood et al. 2008), which directly involves the amino acids leucine, valine, isoleucine, phenylalanine, tyrosine, and tryp- tophan. Thus, nitrogen concentration and relative amino-acid ratios both directly affect the formation of fusel alcohols (Hazelwood et al. 2008). Nitrogen concentration will also affect ester formation, as fusel alcohols are precursors for acetate esters (Saerens et al. 2010). However, most studies investigating a link between nitrogen supplementation and the production of aroma compounds during fermentation have been conducted in synthetic media or model grape juice (Hernandez-Orte et al. 2006, Carrau et al. 2008), which may not reflect the conditions in a more complex starting matrix. Although the genes regulating fusel alcohol and ester production in laboratory
Figure 4 The starting material $(\mathbf{A})$ used in fermentation will affect glucose repression and nitrogen catabolite repression $(\mathbf{B})$ and also derepression (C). Sugar concentration, the types of sugar, and the relative ratios of the different sugars will affect the length of catabolite repression and the flavor compounds produced during fermentation. (B) In highsugar environments, glucose is preferentially taken up by hexose transporters (Hxt) and phosphorylated to glucose-6-phosphate in glycolysis (De Deken 1966). The rise in glucose6-phosphate and ATP in the cell inactivates the Snf1 complex (Wilson et al. 1996). When Snf1 is inactive, Mip1 moves to the nucleus, recruits the repressors Tup1 and Ssn6, and the $M A L$ genes involved in the uptake of alternative carbon sources are blocked (red proteins and metabolites) (De Vit et al. 1997). Extracellular glucose is sensed by a $G$ protein-coupled receptor (Gpr1) and by Gpa2 (not shown), and a rise in glucose-6-phosphate increases Cyr1 activity (Rolland et al. 2000). The signal creates an increase in cAMP, which binds to Bcy1 (of the PKA complex), releasing the Tpk1 catalytic subunits (Toda et al. 1987). Tpk1 phosphorylates several proteins outside the nucleus, and these proteins block the transcription of the stress-responsive element (STRE) genes (e.g., Hsp12 and Hsp104) and enhances acetate ester synthesis (green proteins and metabolites) (Thevelein 1994). In the presence of preferred nitrogen sources, ammonium is taken up into the cell, and specific amino acids (glutamine and glutamate) are taken up preferentially by amino acid permeases. The TOR complex is activated, phosphorylating GIn3, which then recruits the repressor Ure2, effectively blocking amino acid uptake of poorer nitrogen sources (blue proteins and metabolites) (Bertram et al. 2000). TOR1 is linked to carbon metabolism, as it is involved in regulating glucose activation and glycolysis during fermentation (Hardwick et al. 1999) (dashed arrows). (C) In low-glucose environments, Snf1 and Mig1 are phosphorylated, initiating their translocation to the cytoplasm (De Vit et al. 1997). The proteins involved in the uptake of alternative carbon sources (i.e., maltose and maltotriose) are translated (red proteins and enzymes) (De Vit et al. 1997). Additionally, the PKA complex is not activated, allowing expression of the STRE genes (green proteins and enzymes). Low-nitrogen conditions do not trigger activation of the TOR complex, allowing Gln3 to activate multiple nitrogen permeases for nitrogen uptake, including the ammonium permeases Mep1, 2, and 3; Gap1; Agp1 (general amino acid permeases); and Put4 (proline-specific permease) (Beck and Hall 1999). Gln1p and Gdh2p are translated and involved in glutamate and $\alpha$-ketoglutarate synthesis, respectively (blue proteins and enzymes) (Miller and Magasanik 1990, Filetici et al. 1996). The sugar transporters are represented by shades of red; darker red represents a higher sugar-uptake preference. PM: plasma membrane; NM: nuclear membrane.

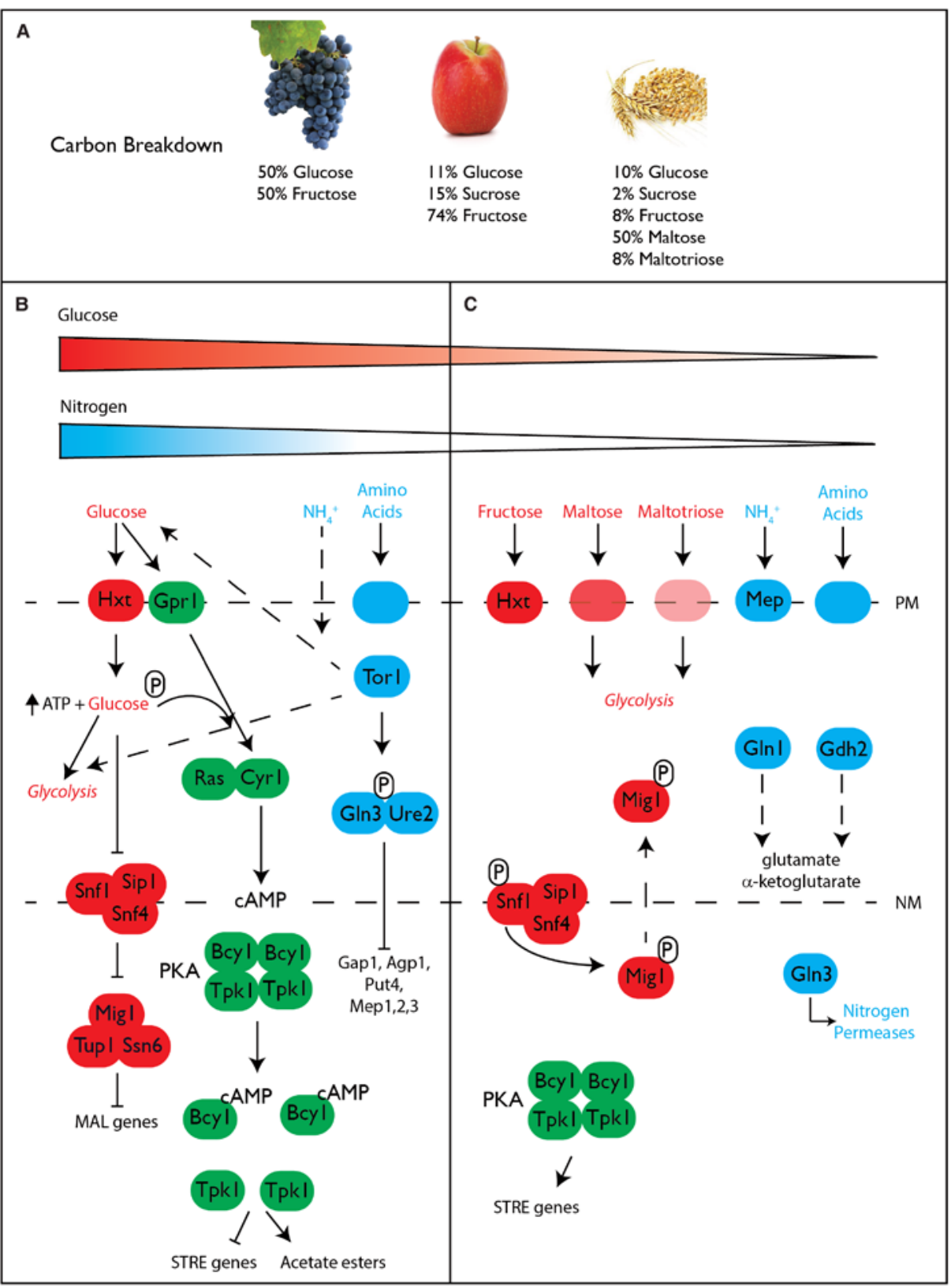

Am. J. Enol. Vitic. 67:4 (2016) 
strains and synthetic media are well known, little is known about nitrogen regulation in commercially relevant strains under production conditions.

\section{Using -Omics Biology to Understand Flavor Production}

Flavor profiles may be optimized during industrial fermentations with approaches that integrate large data sets. In this regard, systems biology often refers to -omics approaches, including genomics, transcriptomics, proteomics, and metabolomics. The data from multiple -omics approaches may be integrated, graphically viewed, and modeled to predict how a particular system functions under a given set of conditions (Ideker et al. 2001). Systems biology methods have been applied to winemaking and brewing to understand how specific genes are linked to specific variations in phenotype and aroma production (Table 1). Directly correlating -omics data with the production of secondary aroma metabolites is a novel approach to improving flavor production in industrial fermentation.

Systems biology approaches have been used to explore the link between yeast gene expression and metabolomics, but most of these studies used the lab strain S288c, which lacks several genes present in wine, ale, and lager yeast strains of S. cerevisiae (Dunn et al. 2005). The genetic dissimilarities between laboratory and industrial strains are due to hybridization, introgression, and variation in gene copy numbers (Dunn et al. 2012). Differences in secondary metabolite composition are observed when industrial strains are used in fermentation, suggesting that genetic differences between lab and industrial strains account for the differences in secondary metabolite production (Howell et al. 2005, Rossouw et al. 2008, Richter et al. 2013). Most transcriptomic studies that have analyzed the impact of environmental changes on yeast fermentation of wines were limited by the use of only a small number of industrially relevant strains (Rossouw et al. 2008, Steyer et al. 2012, García-Ríos et al. 2014). Thus, a broader assessment of commercially relevant $S$. cerevisiae strains is required to fully elucidate the regulation of flavor compounds produced during fermentation.

Table 1 Summary of -omics studies investigating, and approaches applied to, flavor production in Saccharomyces cerevisiae fermentation.

\begin{tabular}{lccc}
\hline & \multicolumn{3}{c}{-Omics approach } \\
\cline { 2 - 4 } Reference & Genomic & Transcriptomic & Metabolomic \\
\hline Howell et al. 2006 & & $\times$ \\
Marullo et al. 2007 & $\times$ & $\times$ & $\times$ \\
Rossouw et al. 2008 & $\times$ & $\times$ \\
Yoshida et al. 2008 & $\times$ & \\
Rossouw and Bauer 2009 & $\times$ & \\
Rossouw et al. 2009 & $\times$ & $\times$ \\
Rossouw et al. 2012 & $\times$ & \\
Celton et al. 2012 & $\times$ \\
Procopio et al. 2014 & & $\times$ \\
Silva Ferreira et al. 2014 & & $\times$ \\
Pinu et al. 2014 & & \\
\hline
\end{tabular}

Transcriptomics studies of $S$. cerevisiae have improved our understanding of the link between gene expression with the production of specific aroma compounds. Many studies have explored genome-wide changes in gene expression with microarray, serial analysis of gene expression, and direct RNA sequencing approaches (James et al. 2003, Rossouw et al. 2008, 2010, Rossouw and Bauer 2009) (Table 1).

Rossouw et al. (2008) analyzed the transcriptome and metabolome profiles of five industrially relevant wine strains to predict the impact of the expression of individual genes on aroma production (Rossouw et al. 2008). Five genes were selected for overexpression in one yeast strain on the basis of a model built from transcriptomic data and the predicted contribution of these genes to the production of higher alcohols and esters. Overexpression of four of the five genes resulted in substantial aroma profile changes that statistically significantly aligned with the aromatic changes predicted by the models. The results of this study illustrate how a systems biology approach can guide genetic manipulation approaches to alter wine-sensory characteristics (Rossouw et al. 2008).

Several -omics approaches have also been applied to brewing to analyze the two types of yeast used for beer: topfermenting $S$. cerevisiae (ale/weiss) or bottom-fermenting $S$. pastorianus (lager) (James et al. 2003, Pham et al. 2006). These studies have been limited by the use of $S$. cerevisiae gene arrays for both ale and lager yeast. Because of the hybrid nature of lager strains (Smart 2007), lager-specific microarray gene chips are required for a more rigorous transcriptomic analysis of lager yeasts.

In brewing studies, global transcription, amino-acid uptake, and production of nine volatile compounds were monitored during wort fermentation with $S$. cerevisiae strain S81 or S. pastorianus strain S23 (Procopio et al. 2014). Both the sequential uptake of amino acids and the aroma profiles differed between the two strains, indicating that these two variables may be linked. The authors suggested that differences in gene transcription, particularly of amino-acid permease genes, affected the uptake of amino acids and the subsequent production of higher alcohols (Procopio et al. 2014). Global transcription patterns coupled with aroma production may help identify genes and metabolites involved in aroma production; however, the hypotheses generated must be tested to confirm a true linkage. For example, juice-manipulation experiments were conducted in Sauvignon blanc must to validate hypotheses generated from a metabolomics study (Pinu et al. 2014). These experiments confirmed the predicted hypotheses and established causal relationships between specific metabolites and the production of 3-MH, 3-MHA, and 4-MMP (Pinu et al. 2014). Confirmation of the links between specific genes and aroma compounds might then be used to build predictive models for altering aroma production during fermentation.

\section{Conclusions}

Here, we describe how secondary metabolism, starting material, carbon and nitrogen profiles, and the fermentation environment all affect the production of secondary metabolites 
produced by $S$. cerevisiae during industrial fermentation. These variables can all be manipulated to produce higher yields of desirable flavor compounds. The power of systems biology may be paired with synthetic-biology approaches to improve the yield and quality of desired secondary metabolites in the cosmetic, perfume, and biofuels industries. In the food and beverage industries, systems biology can be used to map biochemical pathways, but innate genetic variability must be exploited to enhance flavor production. The sheer amount of genomic and experimental data available for $S$. cerevisiae and its long history in industrial fermentation make this organism an ideal target for improving and enhancing its application in biotechnology, particularly for the production of small molecules.

Systems biology approaches applied to $S$. cerevisiae fermentation have focused on differential gene expression (Rossouw and Bauer 2009), correlation of gene and protein expression (Rossouw et al. 2010), differentiating phenotypes of industrial strains (Rossouw et al. 2008, Rossouw and Bauer 2009), and linking gene and protein expression to the production of secondary aroma compounds (Rossouw et al. 2008, Rossouw et al. 2010). Despite this volume of work, we are only beginning to understand how altering the fermentation environment may result in altered secondary metabolism of S. cerevisiae.

The bulk of the literature on systems biology methods applied to fermentation rely heavily on laboratory strains of $S$. cerevisiae; however, many industrial yeast strains are hybrids of $S$. cerevisiae and other Saccharomyces species, or contain genes absent in lab strains. Continued investigation of industrial strains and of non-S. cerevisiae yeasts will uncover genes and pathways responsible for regulating the production of secondary aroma compounds. Ultimately, integration of multiple -omics approaches will lay the foundation for predicting the types and concentrations of flavor-relevant metabolites produced when fermentation conditions are altered. This approach will provide the framework for tailoring industrial fermentations with $S$. cerevisiae to high-quality and high-level production of metabolites across a range of industries.

\section{Literature Cited}

Anderson R and Kirsop B. 1974. The control of volatile ester synthesis during the fermentation of wort of high specific gravity. J I Brewing 80:48-55.

Barker BTP and Burroughs LF. 1953. Cider apple varieties then and now: A survey of vintage-quality trials. In Science and Fruit. T Wallace and RW Marsh (eds.), pp. 45-55. University of Bristol, England.

Beck T and Hall MN. 1999. The TOR signalling pathway controls nuclear localization of nutrient-regulated transcription factors. Nature 402:689-692.

Beech FW. 1972. Cider making and cider research: A review. J I Brewing 78:477-491.

Bertram PG, Choi JH, Carvalho J, Ai W, Zeng C, Chan TF and Zheng XF. 2000. Tripartite regulation of Gln $3 p$ by TOR, Ure2p, and phosphatases. J Biol Chem 275:35727-35733.

Briggs DE, Boulton CA, Brookes PA, and Stevens R. 2004. Brewing: Science and Practice. Woodhead Publishing, Cambridge, and CRA Press, Boca Raton, FL.
Carrau FM, Medina K, Boido E, Farina L, Gaggero C, Dellacassa E, Versini G and Henschke PA. 2005. De novo synthesis of monoterpenes by Saccharomyces cerevisiae wine yeasts. FEMS Microbiol Lett 243:107-115.

Carrau FM, Medina K, Farina L, Boido E, Henschke PA and Dellacassa E. 2008. Production of fermentation aroma compounds by Saccharomyces cerevisiae wine yeasts: Effects of yeast assimilable nitrogen on two model strains. FEMS Yeast Res 8:1196-1207.

Celton M, Sanchez I, Goelzer A, Fromion V, Camarasa C and Dequin S. 2012. A comparative transcriptomic, fluxomic and metabolomic analysis of the response of Saccharomyces cerevisiae to increases in NADPH oxidation. BMC Genomics 13:317.

Conde C, Silva P, Fontes N, Dias ACP, Tavares RM, Sousa MJ, Agasse A, Delrot S and Gerós H. 2007. Biochemical changes throughout grape berry development and fruit and wine quality. Food 1:1-22.

Dale CJ, Young TW and Omole AT. 1990. Small scale mashing experiments with grists containing high proportions of raw sorghum. J I Brewing 96:403-409.

De Deken RH. 1966. The Crabtree effect: A regulatory system in yeast. J Gen Microbiol 44:149-156.

De Vit MJ, Waddle JA and Johnston M. 1997. Regulated nuclear translocation of the Mig1 glucose repressor. Mol Biol Cell 8:1603-1618.

Dufour JP, Malcorps PH and Silcock P. 2008. Control of ester synthesis during brewery fermentation. In Brewing Yeast Fermentation Performance. 2nd ed. K Smart (ed.), pp. 213-233. Blackwell Science, Oxford, UK

Dunn B, Levine RP and Sherlock G. 2005. Microarray karyotyping of commercial wine yeast strains reveals shared, as well as unique, genomic signatures. BMC Genomics 6:53.

Dunn B, Richter C, Kvitek DJ, Pugh T and Sherlock G. 2012. Analysis of the Saccharomyces cerevisiae pan-genome reveals a pool of copy number variants distributed in diverse yeast strains from differing industrial environments. Genome Res 22:908-924.

Filetici P, Martegani MP, Valenzuela L, González A and Ballario P. 1996. Sequence of the GLT1 gene from Saccharomyces cerevisiae reveals the domain structure of yeast glutamate synthase. Yeast 12:1359-1366

García-Ríos E, López-Malo M and Guillamón JM. 2014. Global phenotypic and genomic comparison of two Saccharomyces cerevisiae wine strains reveals a novel role of the sulfur assimilation pathway in adaptation at low temperature fermentations. BMC Genomics 15:1059.

Gil JV, Manzanares P, Genovés S, Vallés S and González-Candelas L. 2005. Over-production of the major exoglucanase of Saccharomyces cerevisiae leads to an increase in the aroma of wine. Int Food Microbiol 103:57-68.

Gunata Z, Bitteur S, Brillouet JM, Bayonove C and Cordonnier R. 1988. Sequential enzymic hydrolysis of potentially aromatic glycosides from grape. Carbohydr Res 184:139-149.

Hardwick JS, Kuruvilla FG, Tong JK, Shamji AF and Schreiber SL. 1999. Rapamycin-modulated transcription defines the subset of nutrient-sensitive signaling pathways directly controlled by the Tor proteins. P Natl Acad Sci USA 96:14866-14870.

Harsch MJ, Benkwitz F, Frost A, Colonna-Ceccaldi B, Gardner RC and Salmon JM. 2013. New precursor of 3-mercaptohexan-1-ol in grape juice: Thiol-forming potential and kinetics during early stages of must fermentation. J Agric Food Chem 61:3703-3713.

Hazelwood LA, Daran JM, van Maris AJ, Pronk JT and Dickinson JR. 2008. The Ehrlich pathway for fusel alcohol production: A century of research on Saccharomyces cerevisiae metabolism. Appl Environ Microbiol 74:2259-2266.

Henschke PA and Jiranek V. 1993. Yeasts-metabolism of nitrogen compounds. In Wine Microbiology and Biotechnology. GH Fleet (ed.), pp. 77-164. Harwood Academic Publishers, Chur, Switzerland. 
Hernandez-Orte P, Bely M, Cacho J and Ferreira V. 2006. Impact of ammonium additions on volatile acidity, ethanol, and aromatic compound production by different Saccharomyces cerevisiae strains during fermentation in controlled synthetic media. Aust J Grape Wine Res 12:150-160.

Howell KS, Klein M, Swiegers JH, Hayasaka Y, Elsey GM, Fleet GH, Høj PB, Pretorius IS and de Barros Lopes MA. 2005. Genetic determinants of volatile-thiol release by Saccharomyces cerevisiae during wine fermentation. Appl Environ Microbiol 71:5420-5426.

Howell KS, Cozzolino D, Bartowsky EJ, Fleet GH and Henschke PA. 2006. Metabolic profiling as a tool for revealing Saccharomyces interactions during wine fermentation. FEMS Yeast Res 6:91-101.

Ideker T, Galitski T and Hood L. 2001. A new approach to decoding life: Systems biology. Annu Rev Genomics Hum Genet 2:343-372.

James T, Campbell S, Donnelly D and Bond U. 2003. Transcription profile of brewery yeast under fermentation conditions. J Appl Microbiol 94:432-448.

Jones M and Pierce JS. 1964. Absorption of amino acids from wort by yeasts. J I Brewing 70:307-315.

King A and Richard Dickinson J. 2000. Biotransformation of monoterpene alcohols by Saccharomyces cerevisiae, Torulaspora delbrueckii and Kluyveromyces lactis. Yeast 16:499-506.

Klose C, Mauch A, Wunderlich S, Thiele F, Zarnkow M, Jacob F and Arendt EK. 2011. Brewing with $100 \%$ oat malt. J I Brewing 117:411-421.

Lambrechts M and Pretorius I. 2000. Yeast and its importance to wine aroma - A review. S Afr J Enol Vit 21:97-129.

Laurent MH, Henick-Kling T and Acree T. 1994. Changes in the aroma and odor of Chardonnay wine due to malolactic fermentation. Wein-Wiss 49:3-10

Lilly M, Bauer FF, Styger G, Lambrechts MG and Pretorius IS. 2006. The effect of increased branched-chain amino acid transaminase activity in yeast on the production of higher alcohols and on the flavour profiles of wine and distillates. FEMS Yeast Res 6:726-743.

Marullo P, Aigle M, Bely M, Masneuf-Pomarède I, Durrens P, Dubourdieu D and Yvert G. 2007. Single QTL mapping and nucleotide-level resolution of a physiologic trait in wine Saccharomyces cerevisiae strains. FEMS Yeast Res 7:941-952.

Miller SM and Magasanik B. 1990. Role of NAD-linked glutamate dehydrogenase in nitrogen metabolism in Saccharomyces cerevisiae. J Bacteriol 172:4927-4935.

Moreno-Arribas MV and Polo C. 2009. Wine Chemistry and Biochemistry. Springer-Verlag, New York.

Pham TK, Chong PK, Gan CS and Wright PC. 2006. Proteomic analysis of Saccharomyces cerevisiae under high gravity fermentation conditions. J Proteome Res 5:3411-3419.

Pinu FR, Edwards PJB, Jouanneau S, Kilmartin PA, Gardner RC and Villas-Boas SG. 2014. Sauvignon blanc metabolomics: Grape juice metabolites affecting the development of varietal thiols and other aroma compounds in wines. Metabolomics 10:556-573.

Procopio S, Brunner M and Becker T. 2014. Differential transcribed yeast genes involved in flavour formation and its associated amino acid metabolism during brewery fermentation. Euro Food Res Technol 239:421-439.

Rauhut D. 1993. Yeasts-production of sulfur compounds. In Wine Microbiology and Biotechnology. GH Fleet (ed.), pp. 183-223. Harwood Academic Publishers, Chur, Switzerland.

Reifenberger E, Boles E and Ciriacy M. 1997. Kinetic characterization of individual hexose transporters of Saccharomyces cerevisiae and their relation to the triggering mechanisms of glucose repression. Eur J Biochem 245:324-233.
Richter CL and Pugh T. 2012. Comparative phenotypic profiling of S. cerevisiae wine, beer and sake yeast strains in Chardonnay wine fermentation. MBAA TQ 49:123-130.

Richter CL, Dunn B, Sherlock G and Pugh T. 2013. Comparative metabolic footprinting of a large number of commercial wine yeast strains in Chardonnay fermentations. FEMS Yeast Res 13:394-410.

Rodríguez JM, Ruíz-Sala P, Ugarte M and Peñalva MÁ. 2004. Fungal metabolic model for 3-methylcrotonyl-CoA carboxylase deficiency. J Biol Chem 279:4578-4587.

Rolland F, De Winde H, Lemaire K, Boles E, Thevelein JM and Winderickx J. 2000. Glucose-induced cAMP signalling in yeast requires both a G-protein coupled receptor system for extracellular glucose detection and a separable hexose kinase-dependent sensing process. Mol Microbiol 38:348-358.

Rossouw D and Bauer FF. 2009. Comparing the transcriptomes of wine yeast strains: Toward understanding the interaction between environment and transcriptome during fermentation. Appl Microbiol and Biotechnol 84:937-954.

Rossouw D, Næs T and Bauer FF. 2008. Linking gene regulation and the exo-metabolome: A comparative transcriptomics approach to identify genes that impact on the production of volatile aroma compounds in yeast. BMC Genomics 9:530.

Rossouw D, Olivares-Hernandes R, Nielsen J and Bauer FF. 2009. Comparative transcriptomic approach to investigate differences in wine yeast physiology and metabolism during fermentation. Appl Environ Microbiol 75:6600-6612.

Rossouw D, van den Dool AH, Jacobson D and Bauer FF. 2010. Comparative transcriptomic and proteomic profiling of industrial wine yeast strains. Appl Environ Microbiol 76:3911-3923.

Rossouw D, Jacobson D and Bauer FF. 2012. Transcriptional regulation and the diversification of metabolism in wine yeast strains. Genetics 190:251-261.

Saerens SM, Verstrepen KJ, Van Laere SD, Voet AR, Van Dijck P, Delvaux FR and Thevelein JM. 2006. The Saccharomyces cerevisiae $E H T 1$ and EEB1 genes encode novel enzymes with medium-chain fatty acid ethyl ester synthesis and hydrolysis capacity. J Biol Chem 281:4446-4456.

Saerens SM, Delvaux FR, Verstrepen KJ and Thevelein JM. 2010. Production and biological function of volatile esters in Saccharomyces cerevisiae. Microb Biotechnol 3:165-177.

Sentheshanmuganathan S. 1960. The mechanism of the formation of higher alcohols from amino acids by Saccharomyces cerevisiae. Biochem J 74:568-576.

Silva Ferreira AC, Monforte AR, Teixeira CS, Martins R, Fairbairn S and Bauer FF. 2014. Monitoring alcoholic fermentation: An untargeted approach. J Agric Food Chem 62:6784-6793.

Smart KA. 2007. Brewing yeast genomes and genome-wide expression and proteome profiling during fermentation. Yeast 24:993-1013.

Spiropoulos A and Bisson LF. 2000. MET17 and hydrogen sulfide formation in Saccharomyces cerevisiae. Appl Environ Microbiol 66:4421-4426.

Steyer D et al. 2012. QTL mapping of the production of wine aroma compounds by yeast. BMC Genomics 13:573.

Styger G, Prior B and Bauer FF. 2011. Wine flavor and aroma. J Ind Microbiol Biotechnol 38:1145-1159.

Suomalainen H and Ronkainen P. 1968. Mechanism of diacetyl formation in yeast fermentation. Nature 220:792-793.

Swiegers JH and Pretorius IS. 2005. Yeast modulation of wine flavor. Adv Appl Microbiol 57:131-175.

Taylor GT and Kirsop BH. 1977. The origin of the medium chain length fatty acids present in beer. J I Brewing 83:241-243. 
Thevelein JM. 1994. Signal transduction in yeast. Yeast 10:1753-1790.

Thomas D and Surdin-Kerjan Y. 1997. Metabolism of sulfur amino acids in Saccharomyces cerevisiae. Microbiol Mol Biol Rev 61:503-532.

Toda T, Cameron S, Sass P, Zoller M and Wigler M. 1987. Three different genes in $S$. cerevisiae encode the catalytic subunits of the cAMP-dependent protein kinase. Cell 50:277-287.

Tominaga T, Peyrot des Gachons C and Dubourdieu D. 1998. A new type of flavor precursors in Vitis vinifera L. cv. Sauvignon blanc: S-cysteine conjugates. J Agric Food Chem 46:5215-5219.

Vandamme EJ and W Soetaert. 2002. Bioflavours and fragrances via fermentation and biocatalysis. J Chem Tech and Biotech 77:1323-1332.

Venkataraman NS, Panneerselvam D, Parija TK, Govindanayagi P and Geetha K. 2014. Global Markets for Flavors and Fragrances. bcc Research Report Code No. CHM034D, Wellesley, MA.

Verstrepen KJ, Van Laere SD, Vanderhaegen BM, Derdelinckx G, Dufour JP, Pretorius IS, Winderickx J, Thevelein JM and Delvaux FR. 2003. Expression levels of the yeast alcohol acetyltransferase genes $A T F 1, \mathrm{Lg}-A T F 1$, and $A T F 2$ control the formation of a broad range of volatile esters. Appl Environ Microbiol 69:5228-5237.

Verstrepen KJ, Iserentant D, Malcorps P, Derdelinckx G, Van Dijck P, Winderickx J, Pretorius IS, Thevelein JM and Delvaux FR. 2004.
Glucose and sucrose: hazardous fast-food for industrial yeast? Trends Biotechnol 22:531-537.

Wilson WA, Hawley SA and Hardie DG. 1996. Glucose repression/ derepression in budding yeast: SNF1 protein kinase is activated by phosphorylation under derepressing conditions, and this correlates with a high AMP:ATP ratio. Curr Biol 6:1426-1434.

Yoshida S et al. 2008. Development of bottom-fermenting Saccharomyces strains that produce high $\mathrm{SO}_{2}$ levels, using integrated metabolome and transcriptome analysis. Appl Environ Microbiol 74:2787-2796.

Yoshimoto H, Fujiwara D, Momma T, Ito C, Sone H, Kaneko Y and Tamai Y. 1998. Characterization of the ATF1 and Lg-ATF1 genes encoding alcohol acetyltransferases in the bottom fermenting yeast Saccharomyces pastorianus. J Ferment Bioeng 86:15-20.

Younis OS and Stewart GG. 1999. Effect of malt wort, very-high-gravity malt wort, and very-high-gravity adjunct wort on volatile production in Saccharomyces cerevisiae. J Am Soc Brew Chem 57:39-45.

Younis OS and Stewart G. 2000. The effect of wort maltose content on volatile production and fermentation performance in brewing yeast. In Brewing Yeast Fermentation Performance. K Smart (ed.), pp. 170-176. Blackwell Science, Oxford. 